\title{
Gesetzestext des Jugendwohlfahrtsgesetzes
}

\author{
Abschnitt I \\ Allgemeines
}

\section{$\S 1$}

(1) Jedes deutsche Kind hat ein Recht auf Erziehung zur leiblichen, seelischen und gesellschaftlichen Tüchtigkeit.

(2) Das Recht und die Pflicht der Eltern zur Erziehung werden durch dieses Gesetz nicht berührt. Gegen den Willen des Erziehungsberechtigten ist ein Eingreifen nur zulässig, wenn ein Gesetz es erlaubt.

(3) Insoweit der Anspruch des Kindes auf Erziehung von der Familie nicht erfüllt wird, tritt, unbeschadet der Mitarbeit freiwilliger Tätigkeit öffentliche Jugendhilfe ein.

\section{$\S 2$}

(1) Organe der öffentlichen Jugendhilfe sind die Jugendwohlfahrtsbehörden (Jugendämter, Landesjugendämter, Reichsjugendamt) ${ }^{*}$ ), soweit nicht gesetzlich die Zuständigkeit anderer öffentlicher Körperschaften oder Einrichtungen, insbesondere der Schule, gegeben ist.

(2) Die öffentliche Jugendhilfe umfaßt alle behördlichen Maßnahmen zur Förderung der Jugendwohlfahrt (Jugendpflege und Jugendfürsorge) und regelt sich, unbeschadet der bestehenden Gesetze nach den folgenden Vorschriften.

\section{Abschnitt II \\ Jugendwohlfahrtsbehörden}

\section{Jugendamt}

a) Zuständigkeit

\section{$\S 3$}

Aufgaben des Jugendamts sind:

1. der Schutz der Pflegekinder gemä $\beta \S 19$ bis 31 ;

2. die Mitwirkung im Vormundschaftswesen, insbesondere die Tätigkeit des Gemeindewaisenrats gemä $\beta \S 32$ bis 48 ;

*) jetzt Bundesjugendamt (noch nicht errichtet)

$1 a^{*}$ 
3. ........

4. die Mitwirkung bei der Schutzaufsicht und der Fürsorgeerziehung gemäß $\S \S 56$ bis 76 ;

5. die Jugendgerichtshilfe gemäß reichsgesetzlicher*) Regelung;

6. die Mitwirkung bei der Beaufsichtigung der Arbeit von Kindern und jugendlichen Arbeitern nach näherer landesrechtlicher vorschrift;

7. die Mitwirkung bei der Fürsorge für Kriegerwaisen und Kinder von Kriegsbeschädigten;

8. die Mitwirkung in der Jugendhilfe bei den Polizeibehörden, insbesondere bei der Unterbringung zur vorbeugenden Verwahrunggemä $B$ näherer landesrechtlicher Vorschrift.

\section{$\S 4$}

(1) Aufgabe des Jugendamts ist ferner, Einrichtungen und Veranstaltungen anzuregen, zu fördern und gegebenenfalls zu schaffen für:

1. Beratung in Angelegenheiten der Jugendlichen;

2. Mutterschutz vor und nach der Geburt;

3. Wohlfahrt der Säuglinge;

4. Wohlfahrt der Kleinkinder;

5. Wohlfahrt der im schulpflichtigen Alter stehenden Jugend außerhalb des Unterrichts;

6. Wohlfahrt der schulentlassenen Jugend.

(2) Das Nähere kann durch die oberste Landesbehörde bestimmt werden.

\section{$\S 5$}

Die Behörden des Reichs**), der Länder, der Selbstverwaltungskörper, die Organe der Versicherungsträger und die Jugendämter haben sich gegenseitig und die Jugendämter einander zur Erfüllung der Aufgaben der Jugendwohlfahrt Beistand zu leisten. Die Organe der Versicherungsträger sind insbesondere zur Auskunfterteilung über alle das Beschäftigungsverhältnis des Minderjährigen und der zu seinem Unterhalt verpflichteten Personen betreffenden Tatsachen verpflichtet. Insoweit finden die Vorschriften des $\S 142$ der Reichsversicherungsordnung, des $\S 346^{* * *}$ ) des Angestelltenversicherungsgesetzes und des § 233 des Reichsknappschaftsgesetzes keine Anwendung.

\section{$\S 6$}

Das Jugendamt hat die freiwillige Tätigkeit zur Förderung der Jugendwohlfahrt unter Wahrung ihrer Selbständigkeit und ihres satzungsmäßigen Charakters zu unterstützen, anzuregen und zur Mitarbeit heranzuziehen, um mit ihr zum Zwecke eines planvollen Ineinandergreifens aller

*) jetzt bundesgeselzilicher

**) jetzt der Bundesrepublik Deutschland

***) jetzt des $\$ 205$ 
Organe und Einrichtungen der öffentlichen und privaten Jugendhilfe und der Jugendbewegung zusammenzuwirken.

\section{$\S 7$}

(1) Das Jugendamt ist zuständig für alle Minderjährigen, die in seinem Bezirk ihren gewöhnlichen Aufenthalt haben. Für vorläufige Maßnahmen ist das Jugendamt zuständig, in dessen Bezirk das Bedürfnis der öffentlichen Jugendhilfe hervortritt.

(2) Streitigkeiten über die Zuständigkeit werden durch die oberste Landesbehörde und, wenn die Jugendämter verschiedenen Ländern angehören, durch das Bundesverwaltungsgericht entschieden.

\section{b) Aufbau und Verfahren}

\section{$\$ 8$}

(1) Die öffentliche Jugendhilfe gemä $ß \S \S 3$ und 4 ist Selbstverwaltungsangelegenheit der Gemeinden und Gemeindeverbände.

(2) Jede kreisfreie Stadt und jeder Landkreis errichten ein Jugendamt.

(3) Die oberste Landesbehörde kann die Errichtung eines gemeinsamen Jugendamtes durch benachbarte Stadt- und Landkreise sowie eines Jugendamtes durch kreisangehörige Gemeindeverbände oder Gemeinden zulassen. Im Bedarfsfalle können in einer Gemeinde mehrere Jugendämter errichtet werden.

\section{\$ 9}

(1) Zusammensetzung, Verfassung und Verfahren des Jugendamtes werden auf Grund landesrechtlicher Vorschriften geregelt.

(2) Das Jugendamt besteht aus dem Jugendwohlfahrtsausschuß und der Verwaltung des Jugendamtes.

(3) Die Aufgaben nach diesem Gesetz werden durch den Jugendwohlfahrtsausschuß und durch die Verwaltung des Jugendamtes wahrgenommen.

\section{$\S 9$ a}

(1) Dem Jugendwohlfahrtsausschuß müssen angehören:

a) Mitglieder der Vertretungskörperschaft und in der Jugendwohlfahrt erfahrene oder tätige Männer und Frauen aller Bevölkerungskreise, die von der Vertretungskörperschaft zu wählen sind;

b) Männer und Frauen, die auf Vorschlag der im Bezirk des Jugendamtes wirkenden Jugendverbände und der freien Vereinigungen der Jugendwohlfahrt durch die Vertretungskörperschaft zu wählen sind. Die freien Vereinigungen und die Jugendverbände haben Anspruch auf $2 / 5$ der Zahl der stimmberechtigten Mitglieder des Ausschusses;

c) der Leiter der Verwaltung oder ein von ihm bestellter Vertreter;

d) der Leiter der Verwaltung des Jugendamtes; 
e) ein Arzt des Gesundheitsamtes;

f) Vertreter der Kirchen und der jüdischen Kultusgemeinde;

g) ein Vormundschaftsrichter oder ein Jugendrichter.

Landesrecht bestimmt, wer die Vertreter zu Buchstaben e und g benennt.

(2) Nach näherer Bestimmung des Landesrechts und der Verfassung des Jugendamtes können weitere Personen dem Jugendwohlfahrtsausschuß angehören.

(3) Stimmberechtigte Mitglieder sind nur die unter Absatz 1 Buchstaben $\mathrm{a}$ und $\mathrm{b}$ aufgeführten Personen. Die übrigen Mitglieder haben nur beratende Stimme. Ob der Leiter der Verwaltung und der Leiter der Verwaltung des Jugendamtes stimmberechtigt sind oder beratend teilnehmen, bestimmt sich nach Landesrecht.

\section{$\S 9$ b}

Der Jugendwohlfahrtsausschuß befaßt sich anregend und fördernd mit den Aufgaben der Jugendwohlfahrt. Er beschließt im Rahmen der von der Vert retungskörperschaft bereitgestellten Mittel, der von ihr erlassenen Satzung und der von ihr gefaßten Beschlüsse über die Angelegenheiten der Jugendhilfe. Er soll in Fragen der Jugendwohlfahrt vor jeder Beschlußfassung der Vertretungskörperschaft gehört werden und hat das Recht, an sie Anträge zu stellen. Er tritt nach Bedarf, zumindest sechsmal im Jahr, zusammen und ist auf Antrag von mindestens einem Drittel der stimmberechtigten Mitglieder einzuberufen.

\section{\$ $9 \mathrm{c}$}

(1) Die laufenden Geschäfte des Jugendamtes werden von dem Leiter der Verwaltung oder in seinem Auftrage von dem Leiter der Verwaltung des Jugendamtes im Rahmen der Satzung und der Beschlüsse der zuständigen Vertretungskörperschaft und des Jugendwohlfahrtsausschusses geführt.

(2) Zum Leiter der Verwaltung des Jugendamtes dürfen nur Personen bestellt werden, die auf Grund ihres Charakters, ihrer Kenntnisse, ihrer Erfahrungen und in der Regel auf Grund einer fachlichen Ausbildung eine besondere Eignung für die Jugendhilfe haben; vor ihrer Bestellung ist der Jugendwohlfahrtsausschuß zu hören.

(3) Für die Wahl und Ausbildung der in der Verwaltung des Jugendamtes auf dem Gebiete der Jugendwohlfahrt tätigen Fachkräfte stellt die oberste Landesbehörde Richtlinien auf und legt die allgemeinen Voraussetzungen für die Eignung fest.

\section{$\$ 10$}

Die den Gesundheitsämtern nach $\S 3$ des Gesetzes über die Vereinheitlichung des Gesundheitswesens v. 3. 7. 1934 (Reichsgesetzbl. I S. 531) übertragenen Aufgaben werden nicht berührt. Das Gesundheitsamt und das Jugendamt müssen ihre Maßnahmen aufeinander abstimmen. 


\section{$\S 11$}

Der Leiter der Verwaltung des Jugendamtes kann im Rahmen der Beschlüsse des Jugendwohlfahrtsausschusses die Erledigung einzelner Geschäfte oder Gruppen von Geschäften besonderen Ausschüssen sowie Vereinigungen für Jugendhilfe, Jugendverbänden oder einzelnen in der Jugendwohlfahrt erfahrenen und bewährten Männern und Frauen widerruflich übertragen. Das Nähere regelt die oberste Landesbehörde, soweit der Bund nicht von seinem Recht gemäß $\S 15$ Gebrauch macht. Die Verpflichtung des Jugendamtes, für die sachgemäße Erledigung der ihm obliegenden Aufgaben Sorge zu tragen, wird hierdurch nicht berührt.

\section{Landesjugendamt}

\section{§ 12}

(1) Zur Sicherung einer gleichmäßigen Erfüllung der den Jugendämtern obliegenden Aufgaben und zur Unterstützung ihrer Arbeit sind Landesjugendämter zu erreichten.

(2) Größere Länder können mehrere Landesjugendämter errichten.

(3) Kleinere Länder können ein gemeinsames Landesjugendamt errichten. Die Jugendämter eines Landes oder eines Landesteils können dem Landesjugendamt eines anderen Landes angeschlossen werden. Auch kann für Jugendämter verschiedener Länder oder Landesteile ein Landesjugendamt erichtet werden.

\section{$\S 13$}

(1) Dem Landesjugendamt liegen ob:

1. die Aufstellung gemeinsamer Richtlinien und die sonstigen geeigneten Maßnahmen für die zweckentsprechende und einheitliche Tätigkeit der Jugendämter seines Bezirks;

2. die Beratung der Jugendämter und die Vermittlung der Erfahrungen auf dem Gebiete der Jugendwohlfahrt;

3. die Schaffung gemeinsamer Veranstaltungen und Einrichtungen für die beteiligten Jugendämter;

4. die Mitwirkung bei der Unterbringung Minderjähriger;

5. die Zusammenfassung aller Veranstaltungen und Einrichtungen, die sich auf die Fürsorge für gefährdete und verwahrloste Minderjährige beziehen;

6. die Mitwirkung bei der Fürsorgeerziehung gemäß $§ 71$;

7. die Vermittlung von Anregungen für die freiwillige Tätigkeit sowie die Förderung der freien Vereinigungen auf allen Gebieten der Jugendwohlfahrt und ihres planmäßigen Zusammenarbeitens untereinander und mit den Jugendämtern im Bereiche des Landesjugendamts; 
8. die Erteilung der Erlaubnis zur Annahme von Pflegekindern durch Anstalten, sowie die Aufsicht über Anstalten gemä $\beta \S 29$.

(2) Weitere Aufgaben können dem Landesjugendamt durch die oberste Landesbehörde übertragen werden.

\section{$\S 14$}

(1) Die Aufgaben des $\S 13$ werden durch den Landesjugendwohlfahrtsausschuß und durch die Verwaltung des Landesjugendamtes im Rahmen der Satzung und der dem Landesjugendamt zur Verfügung gestellten Mittel wahrgenommen.

(2) Die laufenden Geschäfte werden von dem Leiter der Verwaltung des Landesjugendamtes im Rahmen der Satzung und der Beschlüsse des Landesjugendwohlfahrtsausschusses geführt.

(3) Die im Bezirk des Landesjugendamtes wirkenden freien Vereinigungen für Jugendwohlfahrt und die Jugendverbände haben Anspruch auf $2 / 5$ der Zahl der stimmberechtigten Mitglieder des Landesjugendwohlfahrtsausschusses. Sie sind auf Vorschlag der Verbände von der obersten Landesjugendbehörde zu ernennen. Die übrigen Mitglieder werden durch Landesrecht bestimmt.

(4) $\$ 9 \mathrm{c}$ Abs. 2 und 3 gilt entsprechend.

\section{Reichsjugendamt*)}

\section{$\S 15$}

Zur Sicherung einer tunlichst gleichmäßigen Erfüllung der Aufgaben der Jugendämter kann die Bundesregierung mit Zustimmung des Bundesrates Ausführungsvorschriften erlassen.

\section{$\$ 16$}

(1) Bei dem Reichsministerium**) des Innern ist ein Reichsbeirat***) für Jugendwohlfahrt zu errichten. In Verbindung mit ihm bildet die Reichsregierung****) das Reichsjugendamt*). Ihm gehören Vertreter von Landesjugendämtern an. Die Bestimmungen des $\S 9$ Abs. 2 gelten entsprechend.

(2) Dem Reichsjugendamt*) liegt ob, die Bestrebungen auf dem Gebiete der Jugendhilfe zu unterstützen, die Erfahrungen auf dem Gebiete der Jugendwohlfahrt zu sammeln, sie den Landesjugendämtern zu übermitteln, sowie auch sonst für die Verwertung der gesammelten Erfahrungen Sorge zu tragen.

\footnotetext{
*) jetzt Bundesjugendamt (n>ch nicht errichtet)

**) jetzt Bundesministerium

***) jetzt Bundesbeirat

***) jetzt Bundesregierung
} 


\section{$\$ 17$}

Die näheren Bestimmungen über den Aufgabenkreis und über $\mathrm{Zu}-$ sammensetzung, Verfassung und Verfahren des Reichsjugendamts*) werden von der Reichsregierung**) mit Zustimmung des Reichsrats***) erlassen.

\section{Beschwerde}

\section{$\$ 18$}

(1) Das Beschwerderecht gegen Entscheidungen des Jugendamts und des Landesjugendamts regelt sich nach Landesrecht.

(2) Bei Rechtsbeschwerden aus diesem Gesetz entscheidet im letzten Rechtszug das Reichsverwaltungsgericht****). Das Nähere regelt die Reichsregierung**) mit Zustimmung des Reichsrats***).

Abschnitt IIII

\section{Schutz der Pflegekinder}

\section{Erlaubnis zur Annahme}

\section{$\S 19$}

Pflegekinder sind Kinder unter 14 Jahren, die sich dauernd oder nur für einen Teil des Tages, jedoch regelmäßig in fremder Pflege befinden, es sei denn, daß von vornherein feststeht, daß sie unentgeltlich in vorübergehende Bewahrung genommen werden.

\section{$\S 20$}

(1) Wer ein Pflegekind aufnimmt, bedarf dazu der vorherigen Erlaubnis des Jugendamts. In dringenden Fällen ist die nachträgliche Erlaubnis unverzüglich zu bewirken. Wer mit einem solchen Kinde in den Bezirk eines Jugendamts zuzieht, hat die Erlaubnis zur Fortsetzung der Pflege unverzüglich einzuholen.

(2) Steht von vornherein fest, daß ein Kind unentgeltlich oder nicht gewerbsmäßig in vorübergehende Bewahrung genommen wird, so genügt die Anmeldung bei dem Jugendamte.

\footnotetext{
*) jetzt des Bundesjugendamts

*) jetzt Bundesregierung

***) jetzt des Bundesrats

****) jetzt Bundesverwaltungsgericht
} 
(1) Die Bestimmungen dieses Abschnitts finden keine Anwendung, wenn eheliche Kinder bei Verwandten oder Verschwägerten bis zum dritten Grade verpflegt werden, es sei denn, daß diese Personen Kinder entgeltlich, gewerbsmäßig oder gewohnheitsmäßig in Pflege nehmen.

(2) Die Bestimmungen dieses Abschnitts finden ferner keine Anwendung auf Kinder, die aus Anla $B$ auswärtigen Schulbesuchs für einen Teil des Tages in Pflege genommen werden, sowie auf solche Kinder, die zum $Z$ wecke des Schulbesuchs in auswärtigen Schulorten in Familien untergebracht sind, wenn diese von der Leitung der Schule für geeignet erklärt und überwacht sind.

\section{$\S 22$}

(1) Die Voraussetzungen für die Erlaubnis, ihr Erlöschen und ihren Widerruf können nach § 15 oder durch die Landesjugendämter näher bestimmt werden.

(2) Die Erlaubnis kann widerrufen werden, wenn das körperliche, geistige oder sittliche Wohl des Kindes es erfordert.

\section{$\S 23$}

Zuständig für die Erteilung und den Widerruf der Erlaubnis ist das Jugendamt, in dessen Bezirk die Pflegeperson ihren gewöhnlichen Aufenthalt hat.

\section{Aufsicht}

\section{$\S 24$}

(1) Pflegekinder unterstehen der Aufsicht des Jugendamts. Das gleiche gilt für uneheliche Kinder, die sich bei der Mutter befinden.

(2) Die Aufsichtsbefugnisse, insbesondere soweit sie für das gesundheitliche und sittliche Gedeihen des Kindes erforderlich sind, werden nach $\S 15$ oder durch die Landesjugendämter geregelt.

\section{$\S 25$}

(1) Auf Grund von Vorschriften nach § 15 oder von Richtlinien der Landesjugendämter können Pflegekinder durch Anordnung der Jugendämter von der Beaufsichtigung widerruflich befreit werden.

(2) Uneheliche Kinder sollen, solange sie sich bei der Mutter befinden, von der Beaufsichtigung widerruflich befreit werden, wenn das Wohl des Kindes gesichert ist.

(3) Uneheliche Kinder, die gemäß § 1706 Abs. 2 des Bürgerlichen Gesetzbuches den Namen des Ehemanns der Mutter führen, können, solange sie sich bei der Mutter und deren Ehemann in Pflege befinden, wider- 
ruflich von der Beaufsichtigung befreit werden. Das gleiche gilt von Kindern, die bei ihren Großeltern oder ihrem Vorwund verpflegt werden.

\section{$\S 26$}

Wer ein gemäß $\S 24$ Abs. 1 der Aufsicht unterstehendes Kind in Pflege hat, ist verpflichtet, dessen Aufnahme, Abgabe, Wohnungswechsel und Tod dem Jugendamt unverzüglich anzuzeigen. Die näheren Bestimmungen werden nach $\S 15$ oder durch die Landesjugendämter getroffen.

\section{Vorläufige Unterbringung}

\section{$\$ 27$}

(1) Bei Gefahr im Verzuge kann das Jugendamt das Pflegekind sofort aus der Pflegestelle entfernen und vorläufig anderweit unterbringen.

(2) Das Jugendamt ist verpflichtet, das zuständige Vormundschaftsgericht von der erfolgten Wegnahme unverzüglich zu benachrichtigen.

\section{Behördlich angeordnete Familienpflege, Anstalts- und Vereinspflege}

\section{$\S 28$}

Bei Kindern, die von anderen reichs-*) oder landesgesetzlich zuständigen Behörden in Familienpflege untergebracht werden, steht die Erteilung der Erlaubnis und die Aufsicht diesen Behörden zu. Doch kann die Übertragung dieser Befugnisse von diesen Behörden auf das örtlich zuständige Jugendamt durch die zuständige Reichs-**) oder Landesbehörde angeordnet werden.

\section{$\S 29$}

(1) Die Landesjugendämter können Anstalten, die Kinder in Pflege nehmen, von der Anwendung der Bestimmungen der $\S \S 20$ bis 23 widerruflich befreien. Die Befreiung kann nur versagt werden, wenn das Landesjugendamt Tatsachen feststellt, die die Eignung einer Anstalt zur Aufnahme von Pflegekindern ausschließen.

(2) Die Bestimmungen der $\S \S 24$ bis 26 finden mit der Maßgabe Anwendung, daß an die Stelle der Jugendämter die Landesjugendämter treten und die Regelung der Aufsichtsbefugnisse der Landesgesetzgebung vorbehalten bleibt.

(3) Das Landesjugendamt kann bestimmen, inwieweit die Vorschriften dieses Abschnitts auf Pflegekinder, die unter der Aufsicht einer der Jugendwohlfahrt dienenden, von ihm für geeignet erklärten Vereinigung stehen, Anwendung finden.

\footnotetext{
*) jetzt bundes-

**) jetzt Bundes-
} 
(4) Landesrechtlich kann an Stelle der Landesjugendämter die oberste Landesbehörde für zuständig erklärt werden.

\section{Strafbestimmungen}

$\S 30$

(1) Wer ein Pflegekind ohne die vorgeschriebene Erlaubnis oder Anmeldung in Pflege nimmt oder nach Erlöschen oder Widerruf der Erlaubnis in Pflege behält oder wer den gemäß $§ 22$ Abs. 1 erlassenen Vorschriften entgegenhandelt, wird mit Geldstrafe oder mit Haft oder mit Gefängnisstrafe bis zu drei Monaten bestraft.

(2) Die gleiche Strafe trifft denjenigen, der in den nach $\$ 26$ vorgeschriebenen Anzeigen wissentlich unrichtige Angaben macht oder die Leiche eines Pflegekindes oder unehelichen Kindes ohne die vorgeschriebene Anzeige beerdigt.

(3) Wer der in $\S 26$ vorgeschriebenen Anzeigepflicht nicht nachkommt, wird mit Geldstrafe oder mit Haft bestraft.

(4) Die Bestrafung tritt nur auf Antrag des Jugendamts ein. Die Zurücknahme des Antrags ist zulässig.

\section{Ermächtigung für die Landesgesetzgebung}

$\S 31$

Die Befugnis der Landesgesetzgebung, weitere Vorschriften zum Schutze der Kinder zu erlassen sowie Ausnahmen von den Vorschriften der $\S \S 20$ und 24 für die Unterbringung von Kindern in ländlichen Bezirken zuzulassen, bleibt unberührt.

Abschnitt IV

\section{Stellung des Jugendamts im Vormundschaftswesen; Anstalts- und Vereinsvormundschaft}

\section{Amtsvormundschaft}

a) Allgemeine Bestimmungen

\section{$\S 32$}

Das Jugendamt wird Vormund in den durch die folgenden Bestimmungen vorgesehenen Fällen (Amtsvormundschaft). Es kann die Ausübung der vormundschaftlichen Obliegenheiten einzelnen seiner Mitglieder oder Beamten übertragen. Im Umfang der Übertragung sind die Mitglieder und Beamten zur gesetzlichen Vertretung der Mündel befugt. 
Abschnitt IV: Stellung des Jugendamts im Vormundschaftswesen XXIX

\section{$\S 33$}

(1) Auf die Amtsvormundschaft finden die Bestimmungen des Bürgerlichen Gesetzbuchs mit folgender Maßgabe Anwendung. Ein Gegenvormund wird nicht bestellt; dem Amtsvormund stehen die nach $\S \S 1852$ bis 1854 des Bürgerlichen Gesetzbuchs zulässigen Befreiungen zu. Von der Anwendung ausgeschlossen sind die $\S \S 1788,1801,1835,1836$ Abs. 1 Satz 2 bis 4 und Abs. 2, 1837 Abs. 2, 1838, 1844 und 1866.

(2) $\S 1805$ des Bürgerlichen Gesetzbuchs findet mit der Maßgabe Anwendung, daß die Anlegung von Mündelgeld gemäß $§ 1807$ des Bürgerlichen Gesetzbuchs auch bei der das Jugendamt errichtenden Körperschaft zulässig ist. Hat das Jugendamt Aufwendungen zum Zwecke der Führung der Vormindschaft gemacht, so sind ihm diese aus dem Vermögen des Mündels zu ersetzen. Allgemeine Verwaltungskosten werden nicht ersetzt.

(3) Der Amtsvormund hat auf das religiöse Bekenntnis und die Weltanschauung des Mündels oder seiner Familie bei der Unterbringung Rücksicht zu nehmen.

\section{$\S 34$}

Die Landesgesetzgebung kann bestimmen, daß weitere Vorschriften des ersten Titels des dritten Abschnitts im vierten Buche des Bürgerlichen Gesetzbuchs, welche die Aufsicht des Vormundschaftsgerichts in vermögensrechtlicher Hinsicht betreffen, gegenüber dem Amtsvormund außer Anwendung bleiben. Die Prüfung der Schlußrechnung und die Vermittlung ihrer Abnahme durch das Vormundschaftsgericht bleiben hiervon unberührt.

\section{b) Gesetzliche Amtsvormundschaft}

\section{$\S 35$}

(1) Mit der Geburt eines unehelichen Kindes erlangt das Jugendamt des Geburtsorts die Vormundschaft.

(2) Bis zum Eingreifen des zuständigen Vormundschaftsgerichts hat das Amtsgericht des Geburtsorts die erforderlichen vormundschaftsgerichtlichen Maßnahmen zu treffen.

(3) Auf uneheliche Kinder, die im Ausland geboren sind und im Deutschen Reiche*) ihren Aufenthalt nehmen, finden, falls eine Vormundschaft noch nicht eingeleitet ist, die Bestimmungen von Abs. 1 mit der Maßgabe Anwendung, daß das nach $\S 7$ dieses Gesetzes zuständige Jugendamt die Vormundschaft erlangt.

\section{$\S 36$}

Der Standesbeamte hat die nach $\S 48$ des Reichsgesetzes über die Angelegenheiten der freiwilligen Gerichtsbarkeit vom 17./20. Mai 1898

*) jetzt im Gebiet der Bundesrepublik Deutschland 
(Reichsgesetzbl. S. 189/771) dem Vormundschaftsgericht zu erstattende Anzeige über die Geburt eines unehelichen Kindes dem Jugendamt zu übersenden. Dieser Anzeige ist eine Mitteilung über das religiöse Bekenntnis anzufügen. Das Jugendamt hat unter Weiterreichung der Geburtsanzeige den Eintritt der Vormundschaft (§ 35) dem Vormundschaftsgericht unverzüglich anzuzeigen.

\section{$\S 37$}

Das Vormundschaftsgericht hat dem Jugendamt unverzüglich eine Bescheinigung über den Eintritt der Vormundschaft zu erteilen, die bei Beendigung der Vormundschaft zurückzugeben ist.

\section{$\S 38$}

Auf Antrag des Jugendamts oder einer unverheirateten Mutter kann für eine Leibesfrucht ein Pfleger bestellt werden, auch wenn die Voraussetzung des $\S 1912$ Satz 1 des Bürgerlichen Gesetzbuchs nicht gegeben ist. Der Pfleger wird mit der Geburt des Kindes im Einverständnis mit dem Jugendamt Vormund. In diesem Falle findet $§ 35$ keine Anwendung. die Vormundschaft wird bei dem Vormundschaftsgericht geführt, bei dem die Pflegschaft anhängig war.

\section{$\$ 39$}

(1) Sobald es das Wohl des Mündels erfordert, soll das die Vormundschaft führende Jugendamt bei dem Jugendamt eines anderen Bezirks die Weiterführung der Vormundschaft beantragen. Der Antrag kann auch von dem Jugendamt eines anderen Bezirks, sowie von der Mutter und von einem jeden, der ein berechtigtes Interesse des Mündels geltend macht, gestellt werden. Das die Vormundschaft abgebende Jugendamt hat den Übergang dem Vormundschaftsgericht unverzüglich anzuzeigen.

(2) Gegen die Ablehnung des Antrags kann das Vormundschaftsgericht angerufen werden.

\section{$\$ 40$}

Das Vormundschaftsgericht hat das Jugendamt auf seinen Antrag als Amtsvormund zu entlassen und einen Einzelvormund zu bestellen, soweit dies dem Wohle des Mündels nicht entgegensteht.

\section{c) Bestellte Amtsvormundschaft}

\section{$\S 41$}

(1) Das Jugendamt kann unter den Voraussetzungen des $\S 1773$ des Bürgerlichen Gesetzbuchs mit seinem Einverständnis vor den im § 1776 des Bürgerlichen Gesetzbuchs als Vormünder berufenen Personen zum Vormund für einen Minderjährigen bestellt werden, soweit nicht ein geeigneter anderer Vormund vorhanden ist. 
Abschnitt IV: Stellung des Jugendamts im Vormundschaftswesen

(2) Auf die bestellte Amtsvormundschaft finden die $\$ \$ 1789$ und 1791 des Bürgerlichen Gesetzbuchs keine Anwendung. Die Bestellung erfolgt durch schriftliche Verfügung des Vormundschaftsgerichts.

\section{Stellung des Jugendamts zum Vormundschaftsgericht und zur Einzelvormundschaft}

\section{$\S 42$}

(1) Das Jugendamt ist Gemeindewaisenrat. § 11 gilt entsprechend.

(2) Die Landesgesetzgebung kann örtliche Einrichtungen zur Unterstützung des Jugendamts in den. Geschäften des Gemeindewaisenrats treffen.

\section{$\S 43$}

(1) Das Jugendamt hat das Vormundschafstgericht bei allen Maßnahmen zu unterstützen, welche die Sorge für die Person Minderjähriger betreffen, insbesondere durch Begutachtung bei der Festsetzung von Geldrenten für den Unterhalt Minderjähriger. Vor Entscheidungen in den Fällen des $\S 1635$ Abs. 1 S. $2^{*}$ ), des $\S 1666$, des $\S 1727$, des $\S 1728$ Abs. 2, des $\S 1729$ Abs. 2, des $\S 1750$ Abs. 1 und des $\S 1751$ Abs. 2 des Bürgerlichen Gesetzbuchs muß das Vormundsćhaftsgericht das zuständige Jugendamt hören. Bei Gefahr im Verzuge kann das Vormundschaftsgericht einstweilige Anordnungen auch schon vor Anhörung des Jugendamts treffen. Es kann das Jugendamt mit der Ausführung der Anordnungen aus $\S 1631$ Abs. $2, \S 1636$ S. $2^{* *}$ ) des Bürgerlichen Gesetzbuchs und sonstiger Anordnungen mit dessen Einverständnis betrauen.

(2) Das Landesjugendamt kann auf Antrag des Jugendamts Mitglieder oder Beamte des Jugendamts ermächtigen, Beurkundungen gemäB $\S 1718$ und $\S 1720$ Abs. 2 des Bürgerlichen Gesetzbuchs vorzunehmen, sowie die im $\S 1706$ Abs. 2 des Bürgerlichen Gesetzbuchs bezeichneten Erklärungen entgegenzunehmen und $\mathrm{zu}$ beglaubigen.

\section{$\S 44$}

(1) Das Jugendamt soll die Bestellung einer Einzelperson als Vormund beantragen, wenn dies dem Interesse des Mündels förderlich erscheint. Es kann auch die Bestellung eines Mitvormundes für einen bestimmten Wirkungskreis beantragen.

(2) Die Bestellung kann von einem jeden, der ein berechtigtes Interesse des Mündels geltend macht, und von diesem selbst, wenn es das 14. Lebensjahr vollendet hat, beantragt werden. Sie kann auch von Amts wegen erfolgen. Vor der Entscheidung soll das Vormundschaftsgericht das Jugendamt und tunlichst die Mutter des Mündels hören.

\footnotetext{
*) jetzt § 74 EheG.

**) jetzt $\S 75$ EheG.
} 


\section{$\S 45$}

Das Jugendamt hat die Vormünder, Beistände und Pfleger seines Bezirks planmäßig zu beraten und bei der Ausübung ihres Amtes zu unterstützen. Die näheren Bestimmungen hierüber werden nach $\S \mathbf{1 3}$ Abs. 1 Nr. 1 und $\S 15$ getroffen. $\S 11$ gilt entsprechend.

\section{Mitvormundschaft, Gegenvormundschaft, Pflegschaft und Beistandschaft des. Jugendamts}

\section{$\S 46$}

Die vorstehenden Bestimmungen gelten entsprechend für die Bestellung des Jugendamts zum Mitvormund, Gegenvormund, Pfleger oder Beistand und für die Übertragung einzelner Rechte und Pflichten eines Vormunds auf das Jugendamt.

\section{Anstalts- und Vereinsvormundschaft}

\section{$\S 47$}

(1) Vorstände von Anstalten, die unter der Verwaltung des Staates oder einer öffentlichen Körperschaft stehen, sowie Vorstände solcher privaten Anstalten oder Vereine, die vom Landesjugendamt für geeignet erklärt sind, können auf ihren Antrag zu Vormündern bestellt werden (Anstalts- oder Vereinsvormundschaft). Auch können sie zu Pflegern oder Beiständen bestellt werden. Ebenso können ihnen einzelne Rechte und Pflichten des Vormundes übertragen werden. Das Jugendamt muß in den Fällen, in denen der Minderjährige von ihm bevormundet oder versorgt ist, vorher gehört werden.

(2) Auf die Anstalts- und Vureinsvormundschaft finden die Bestimmungen der $\S \S 33,40,41$ und 44 mit der Maßgabe Anwendung, da $B$ ein Gegenvormund bestellt werden kann. Insbesondere ist die Bestellung eines Jugendamts zum Gegenvormunde zulässig.

\section{$\S 48$}

(1) Artikel 136 des Einführungsgesetzes zum Bürgerlichen Gesetzbuch und die $\S \S 1783,1887$ des Bürgerlichen Gesetzbuchs werden aufgehoben. Dem $\S 1784$ des Bürgerlichen Gesetzbuchs wird folgender Abs. 2 angefügt: „Diese Erlaubnis darf nur versagt werden, wenn ein wichtiger dienstlicher Grund vorliegt."

(2) Dem $\$ 1786$ Nr. 1 des Bürgerlichen Gesetzbuchs werden die Worte hinzugefügt: ,,welche zwei und mehr noch nicht schulpflichtige Kinder besitzt oder glaubhaft macht, daß die ihr obliegende Fürsorge für ihre Familie die Ausübung des Amtes dauernd besonders erschwert". 
Abschnitt V

\title{
Die öffentliche Unterstützung hilfsbedürftiger Minderjähriger*)
}

\author{
Abschnitt VI \\ Die Schutzaufsicht und die Fürsorgeerziehung
}

1. Die Schutzaufsicht

\section{$\S 56$}

Ein Minderjähriger ist unter Schutzaufsicht zu stellen, wenn sie zur Verhütung seiner körperlichen, geistigen oder sittlichen Verwahrlosung geboten und ausreichend erscheint.

\section{$\S 57$}

(1) Das Vormundschaftsgericht ordnet die Schutzaufsicht von Amts wegen oder auf Antrag an. Antragsberechtigt sind die Eltern, der gesetzliche Vertreter und das Jugendamt. Das Vormundschaftsgericht muß das Jugendamt vor der Entscheidung über die Schutzaufsicht hören.

(2) Die Entscheidung des Vormundschaftsgerichts ist den in Abs. 1 Genannten und dem Minderjährigen, wenn er das 14. Lebensjahr vollendet hat, bekanntzugeben, soweit ihr Inhalt nach dem Ermessen des Vormundschaftsgerichts ihm ohne erziehlichen Nachteil mitgeteilt werden kann.

(3) Ist das Vormundschaftsgericht nicht das des gewöhnlichen Aufenthaltsorts des Minderjährigen, so soll auf Antrag des Jugendamts die Abgabe an dieses Gericht gemä $B$ § 46 des Reichsgesetzes über die Angelegenheiten der freiwilligen Gerichtsbarkeit stattfinden, sofern nicht besondere Gründe dagegen sprechen.

\section{$\S 58$}

(1) Die Schutzaufsicht besteht in dem Schutze und der Überwachung des Minderjährigen. Derjenige, der die Schutzaufsicht ausübt (Helfer), hat den Erziehungsberechtigten bei der Sorge für die Person des Minderjährigen zu unterstützen und zu überwachen. Die Schutzaufsicht umfaßt die Sorge über das Vermögen nur, insoweit der Arbeitsverdienst des Minderjährigen in Betracht kommt.

*) ersetzt durch RFV und RGr dazu.

1b Riedel, JWG. 
(2) Der Helfer kann für alle Angelegenheiten, für gewisse Arten von Angelegenheiten oder für einzelne Angelegenheiten bestellt werden.

(3) Úber den Umfang seines Wirkungskreises entscheidet die Bestellung.

(4) Der Helfer hat bei der Ausübung seines Amtes das Recht auf $\mathrm{Zu}-$ tritt zu dem Minderjährigen. Die Eltern, der gesetzliche Vertreter und die Personen, denen der Minderjährige zur Verpflegung und Erziehung übergeben ist, sind verpflichtet, dem Helfer Auskunft zu geben.

(5) Der Helfer hat dem Vormundschaftsgericht jeden Fall, in dem es zum Einschreiten berufen ist, unverzüglich anzuzeigen.

\section{$\S 59$}

Die Schutzaufsicht erlischt mit der Volljährigkeit des Minderjährigeit oder durch die rechtskräftige Anordnung der Fürsorgeerziehung. Sie ist aufzuheben, wenn ihr Zweck erreicht oder die Erreichung anderweitig sichergestellt ist.

\section{$\S 60$}

(1) Die Ausübung der Schutzaufsicht wird vom Vormundschaftsgerichte dem Jugendamt oder nach Anhörung des Jugendamts einer Vereinigung für Jugendhilfe oder einer einzelnen Person, soweit die beiden letzteren zur Übernahme der Schutzaufsicht bereit sind, übertragen. Bei der Übertragung ist auf das religiöse Bekenntnis oder die Weltanschauung des Minderjährigen tunlichst Rücksicht zu nehmen. Das Vormundschaftsgericht hat den Helfer zu entlassen, wenn dies dem Wohle des Minderjährigen förderlich erscheint. Die näheren Bestimmungen über die Ausübung werden von der Reichsregierung*) mit Zustimmung des Reichsrats**) oder von der obersten Landesbehörde getroffen.

(2) Über die Führung des unter Schutzaufsicht gestellten Minderjährigen ist dem Vormundschaftsgericht auf Verlangen Bericht zu erstatten.

(3) Das Jugendamt kann die Schutzaufsicht ohne gerichtliche Anordnung ausüben, solange der Erziehungsberechtigte damit einverstanden ist; es hat in diesem Falle das Vormundschaftsgericht von dem Eintritt der Schutzaufsicht zu benachrichtigen.

\section{\$61}

Eine zur Zeit der Anordnung der Schutzaufsicht bestehende Beistandschaft ( $\S \S 1687 \mathrm{ff}$. des Bürgerlichen Gesetzbuchs) soll insoweit aufgehoben werden, als sich ihr Wirkungskreis mit dem der Schutzaufsicht deckt.

\footnotetext{
*) jetzt Bundesregierung

**) jetzt des Bundesrats
} 


\section{Die Fürsorgeerziehung}

\section{$\$ 62$}

Die Fürsorgeerziehung dient der Verhütung oder Beseitigung der Verwahrlosung und wird in einer geeigneten Familie oder Erziehungsanstalt unter öffentlicher Aufsicht und auf öffentliche Kosten durchgeführt.

\section{$\$ 63$}

(1) Ein Minderjähriger, der das 18. Lebensjahr noch nicht vollendet hat, ist durch Beschluß des Vormundschaftsgerichts der Fürsorgeerziehung zu überweisen.

1. wenn die Voraussetzungen des $\S 1666$ oder des $\S 1838$ des Bürgerlichen Gesetzbuchs vorliegen und zur Verhütung der Verwahrlosung des Minderjährigen die anderweite Unterbringung erforderlich ist, eine nach dem Ermessen des Vormundschaftsgerichts geeignete Unterbringung aber ohne Inanspruchnahme öffentlicher Mittel nicht erfolgen kann. Zur Verhütung lediglich körperlicher Verwahrlosung ist die Überweisung nicht zulässig;

2. wenn die Fürsorgeerziehung zur Beseitigung der Verwahrlosung wegen Unzulänglichkeit der Erziehung erforderlich ist.

(2) Die Fürsorgeerziehung darf nicht angeordnet werden, wenn sie offenbar keine Aussicht auf Erfolg bietet.

(3) Für den Fall, daß Aussicht auf Erfolg der Fürsorgeerziehung besteht, kann diese mit Zustimmung der Fürsorgeerziehungsbehörde auch noch angeordnet werden, wenn der Minderjährige das 18. Lebensjahr, aber noch nicht das 19 . Lebensjahr vollendet hat.

(4) Maßgebend für die Altersgrenze ist der Zeitpunkt, in dem der Antrag bei Gericht eingeht oder das Verfahren gemäß $\S 65$ oder $\S 67$ eingeleitet wird; der Zeitpunkt ist aktenkundig zu machen.

\section{$\S 64$}

Artikel 135 des Einführungsgesetzes zum Bürgerlichen Gesetzbuch wird aufgehoben.

\section{§ 65}

(1) Das Vormundschaftsgericht beschließt von Amts wegen oder auf Antrag. Antragsberechtigt ist das nach $\S 7$ zuständige Jugendamt. Das Antragsrecht kann landesgesetzlich ausgedehnt werden.

(2) Das Vormundschaftsgericht muB vor der Beschlußfassung das Jugendamt, es soll, soweit dies ohne erhebliche Schwierigkeiten geschehen kann, den Minderjährigen, seine Eltern und seinen gesetzlichen Vertreter hören; weitere Anhörungen kann die Landesgesetzgebung vorschreiben. 
(3) Der Beschluß ist mit Gründen zu versehen und muß, wenn er auf Anordnung der Fürsorgeerziehung lautet, den Eintritt der gesetzlichen Voraussetzungen unter Bezeichnung der für erwiesen erachteten Tatsachen feststellen.

(4) Das Vormundschaftsgericht kann die ärztliche Untersuchung des Minderjährigen anordnen und auf die Dauer von höchstens sechs Wochen ihn in einer zur Aufnahme von jugendlichen Psychopathen geeigneten Anstalt oder in einer öffentlichen Heil- und Pflegeanstalt zur Beobachtung unterbringen lassen.

(5) Der die Fürsorgeerziehung anordnende Beschlu $B$ ist den Antragsberechtigten, dem gesetzlichen Vertreter, den Eltern, der Fürsorgeerziehungsbehörde und ferner dem Minderjährigen selbst, wenn er das 14. Lebensjahr vollendet hat und insoweit sein Inhalt nach dem Ermessen des Vormundschaftsgerichts ihm ohne erziehlichen Nachteil mitgeteilt werden kann, zuzustellen. Der die Fürsorgeerziehung ablehnende Beschluß ist dem Antragsteller, der Fürsorgeerziehungsbehörde und, wenn eine vorläufige Fürsorgeerziehung ( $\$ 67$ ) angeordnet ist, ferner allen Personen zuzustellen, denen diese Anordnung zugestellt ist.

(6) Gegen den Beschluß steht die sofortige Beschwerde mit aufschiebender Wirkung den Antragsberechtigten, der Fürsorgeerziehungsbehörde und, wenn der BeschluB auf Fürsorgeerziehung lautet, ferner dem gesetzlichen Vertreter, den Eltern und dem Minderjährigen zu, wenn er das 14. Lebensjahr vollendet hat.

(7) Ist das Vormundschaftsgericht nicht das des gewöhnlichen Aufenthaltsorts des Minderjährigen, so soll auf Antrag des Jugendamts die Abgabe an dieses Gericht gemäß § 46 des Reichsgesetzes über die Angelegenheiten der freiwilligen Gerichtsbarkeit stattfinden, sofern nicht besondere Gründe dagegen sprechen.

\section{$\S 66$}

(1) Das Fürsorgeerziehungsverfahren kann durch Beschluß des Vormundschaftsgerichts auf längstens ein Jahr ausgesetzt werden. Die Aussetzung kann aus besonderen Gründen durch Beschluß des Vormundschaftsgerichts auf höchstens ein weiteres Jahr verlängert werden. Über das vollendete 19. Lebensjahr hinaus kann das Verfahren nicht ausgesetzt werden.

(2) Gegen die Aussetzung steht dem Jugendamt und der Fürsorgeerziehungsbehörde das Recht der sofortigen Beschwerde zu.

(3) Für die Dauer der Aussetzung muß eine Schutzaufsicht gemäß $\S \S 56 \mathrm{ff}$. angeordnet werden.

\section{$\S 67$}

Bei Gefahr im Verzuge kann das Vormundschaftsgericht die vorläufige Fürsorgeerziehung des Minderjährigen beschließen. Das Vormundschaftsgericht kann die vorläufige Fürsorgeerziehung auch zur Prüfung, ob die 
Fürsorgeerziehung Aussicht auf Erfolg bietet, beschließen. Gegen den Beschluß steht den im $\S 65$ Abs. 6 Genannten die sofortige Beschwerde zu. $\S 18$ Abs. 2 des Reichsgesetzes über die Angelegenheiten der freiwilligen Gerichtsbarkeit findet keine Anwendung.

\section{$\S 68$}

(1) Für schleunige, auf Grund dieses Abschnitts zu treffende Maßregeln, ist neben dem im $\S 43$ des Reichsgesetzes über die Angelegenheiten der freiwilligen Gerichtsbarkeit bezeichneten Gericht einstweilen auch dasjenige Gericht zuständig, in dessen Bezirk das Bedürfnis der Fürsorge hervortritt. Das Gericht hat von der angeordneten Maßregel dem endgültig und nunmehr ausschließlich zuständigen Gerichte Mitteilung zu machen.

(2) § 43 Abs. 2 des Reichsgesetzes über die Angelegenheiten der freiwilligen Gerichtsbarkeit findet auch Anwendung, wenn über die Person, in Ansehung deren eine Verrichtung des Vormundschaftsgerichts erforderlich wird, eine Schutzaufsicht oder ein Fürsorgeerziehungsverfahren anhängig ist.

\section{$\$ 69$}

(1) Im Falle der Familienerziehung ist der Minderjährige mindestens bis zum Aufhören der Schulpflicht in einer Familie seines Bekenntnisses, im Falle der Anstaltserziehung soweit möglich, in einer Anstalt seines Bekenntnisses unterzubringen.

(2) Minderjährige ohne Bekenntnis sollen nur mit ihrem Einverständnis, sofern sie ihr Bekenntnis selbst bestimmen können, andernfalls mit demjenigen des Erziehungsberechtigten in einer Familie oder in einer Anstalt eines bestimmten Bekenntnisses untergebracht werden.

(3) Den Erziehungsberechtigten muß von dem Ort der Unterbringung des Kindes sofort Mitteilung gemacht werden, sofern dadurch der Erziehungszweck nicht ernstlich gefährdet wird. Gegen eine Verweigerung dieser Mitteilung steht den Erziehungsberechtigten das Recht der Beschwerde an das Vormundschaftsgericht zu.

(4) In Ausführung einer angeordneten Fürsorgeerzièhung kann die Erziehung in der eigenen Familie der Minderjährigen unter öffentlicher Aufsicht widerruflich angeordnet werden, wenn dadurch die Erreichung des Zweckes der Fürsorgeerziehung nicht gefährdet wird. Innerhalb der ersten drei Monate nach Ausführbarkeit des Fürsorgeerziehungsbeschlusses bedarf die Anordnung der Zustimmung des Vormundschaftsgerichts. Gegen die Verweigerung der Zustimmung steht der Fürsorgeerziehungsbehörde die sofortige Beschwerde zu.

\section{$\$ 70$}

(1) Die Landesgesetzgebung regelt die Ausführung der Fürsorgeerziehung und bestimmt die Fürsorgeerziehungsbehörde, sowie die Träger der Kosten. Nach Möglichkeit ist die Fürsorgeerziehungsbehörde mit dem 
Landesjugendamte zu vereinigen. Die durch die vorläufige Fürsorgeerziehung entstehenden Kosten fallen dem für die endgültige Anordnung der Fürsorgeerziehung zuständigen Kostenträger auch dann zur Last, wenn die Fürsorgeerziehung endgültig nicht angeordnet wird. Besteht über den Ersatz der Kosten zwischen den Fürsorgeerziehungsbehörden für den gewöhnlichen und vorübergehenden Aufenthalt Streit, so gilt $\S 7$ Abs. 2 entsprechend.

(2) Eine von dem zuständigen Vormundschaftsgericht angeordnete Fürsorgeerziehung eines Minderjährigen muß von der Fürsorgeerziehungsbehörde des Orts, der die Zuständigkeit begründet hat, ausgeführt werden. Sie soll regelmäßig sich bei der Ausführung der Fürsorgeerziehung der Jugendämter bedienen. Die Ausführbarkeit der Fürsorgeerziehung tritt mit der Rechtskraft, bei der vorläufigen Fürsorgeerziehung mit dem Erlasse des Beschlusses ein. Die Unterbringung soll unter ärztlicher Mitwirkung erfolgen.

(3) Die Fürsorgeerziehungsbehörde gilt für alle Rechtsgeschäfte, die die Eingehung, Änderung oder Aufhebung eines Dienst- oder Lehrvertrages oder die Geltendmachung der sich aus einem solchen Vertrage ergebenden Ansprüche betreffen, als gesetzliche Vertreterin des Minderjährigen. Sie ist insbesondere befugt, den Arbeitsverdienst des Minderjährigen zu verwalten und für ihn zu verwenden.

(4) Die Fürsorgeerziehungsbehörde ist befugt, die Entmündigung eines Fürsorgezöglings wegen Geisteskrankheit oder Geistesschwäche $\mathrm{zu}$ beantragen.

\section{$\S 71$}

Das Landesjugendamt ist, soweit es nicht selbst Fürsorgeerziehungsbehörde ist, nach näherer Bestimmung der Landesgesetzgebung bei der Ausführung der Fürsorgeerziehung zu beteiligen; es soll insbesondere bei dem Erla $\beta$ allgemeiner, grundsätzlicher Anordnungen über die Art ihrer Ausführung gutachtlich gehört werden und ist zu Vorschlägen über die Ausführung befugt; ihm kann ferner die Mitwirkung bei wichtigen Maßnahmen der Fürsorgeerziehungsbehörde und bei der Aufsicht über die in Anstalten seines Bezirks untergebrachten Zöglinge sowie die Zuständigkeit zur Entscheidung von Beschwerden über Anordnungen der Fürsorgeerziehungsbehörde, die die Ausführung betreffen, übertragen werden, səfern dafür nicht die Gerichte für zuständig erklärt werden.

\section{$\S 72$}

(1) Die Fürsorgeerziehung endigt mit der Vollendung des 19. Lebensjahres.

(2) Die Fürsorgeerziehung ist früher aufzuheben, wenn ihr Zweck erreicht oder anderweitig sichergestellt ist, und zwar von Amts wegen oder auf Antrag der in $\S 65$ Abs. 6 Genannten mit Ausnahme des Minderjährigen. Die Aufhebung kann auch unter Vorbehalt des Widerrufs erfolgen, dessen Ausübung landesgesetzlich zu regeln ist. 
Abschnitt VI: Die Schutzaufsicht und die Fürsorgeerziehung XXXIX

(3) Landesgesetzlich kann bestimmt werden, daß für die Entscheidung über die Aufhebung gemäß Abs. 2 das Vormundschaftsgericht oder die Fürsorgeerziehungsbehörde zuständig ist mit der Maßgabe, daß der Anitragsteller, wenn die Fürsorgeerziehungsbehörde zuständig ist und die Aufhebung ablehnt, binnen zwei Wochen seit Zustellung des ablehnenden Beschlusses die Entscheidung des Vormundschaftsgerichts anrufen kann, gegen dessen BeschluB die sofortige Beschwerde stattfindet. Sofern das Vormundschaftsgericht für die Aufhebung der Fürsorgeerziehung zuständig ist, muß es vor seiner Entscheidung die Fürsorgeerziehungsbehörde gutachtlich hören; dieser steht gegen den die Fürsorgeerziehung aufhebenden Beschluß die sofortige Beschwerde mit aufschiebender Wirkung zu.

(4) Der Antrag auf Aufhebung kann außer vom Jugendamt nicht vor Ablauf eines Jahres seit der Rechtskraft des die Fürsorgeerziehung anordnenden Beschlusses gestellt, ein abgewiesener Antrag kann vor dem Ablauf von sechs Monaten nicht erneuert werden.

\section{$\S 72 \mathrm{a}$}

(1) Auf Antrag der Fürsorgeerziehungsbehörde kann beim Vorliegen besonderer Verhältnisse die Fortführung der Fürsorgeerziehung vom Vormundschaftsgericht über das vollendete 19. Lebensjahr, jedoch nicht über den Zeitpunkt des Eintritts der Volljährigkeit des Minderjährigen hinaus angeordnet werden, wenn die Fortführung zur Erreichung des Zweckes der Fürsorgeerziehung erforderlich ist. Das Antragsrecht der Fürsorgeerziehungsbehörde kann von der obersten Landesbehörde auf bestimmte Fälle beschränkt oder an das Vorliegen bestimmter Voraussetzungen geknüpft werden.

(2) Die Anhörung des Minderjährigen, des gesetzlichen Vertreters und der Eltern steht im Ermessen des Vormundschaftsgerichts. Der Beschluß ist dem Minderjährigen, dem gesetzlichen Vertreter und den Eltern mitzuteilen; im Falle der Ablehnung erfolgt die Mitteilung an die Fürsorgeerziehungsbehörde durch Zustellung.

(3) Das Vormundschaftsgericht entscheidet endgültig; doch steht der Fürsorgeerziehungsbehörde gegen den die Fortführung der Fürsorgeerziehung ablehnenden Beschluß die sofortige Beschwerde zu.

(4) Solange über den Antrag nicht rechtskräftig entschieden ist, dauert die Fürsorgeerziehung fort.

\section{$\S 73$}

Die Fürsorgeerziehungsbehörde kann einen Minderjährigen nach Vollendung des 18. Lebensjahres wegen Unausführbarkeit der Fürsorgeerziehung aus Gründen, die in der Person des Minderjährigen liegen, nach Anhörung des Jugendamts aus der Fürsorgeerziehung unter der Voraussetzung entlassen, daß die Fürsorgeerziehung mindestens ein Jahr gedauert hat. Die Vollendung des 18. Lebensjahres und die einjährige 
Durchführung der Fürsorgeerziehung ist für die Entlassung nicht erforderlich, wenn der Minderjährige an erheblichen geistigen oder seelischen Regelwidrigkeiten leidet. Die Fürsorgeerziehungsbehörde bedarf für die Entlassung der Zustimmung des Vormundschaftsgerichts. Gegen die Versagung der Zustimmung steht der Fürsorgeerziehungsbehörde die sofortige Beschwerde zu.

\section{$\$ 74$}

Die gerichtlichen Verhandlungen sind gebühren- und stempelfrei; die baren Auslagen fallen der Staatskasse zur Last. Die nach $\S 65$ Abs. $2 \mathrm{zu}$ hörenden Personen können, im Falle ihrer Vernehmung vor Gericht, Ersatz ihrer Auslagen nach den für Zeugen geltenden Vorschriften verlangen. Dies gilt jedoch nicht für den Minderjährigen und seine Eltern. Verträge über die Unterbringung von Minderjährigen zur Ausführung dèr Fürsorgeerziehung sind stempelfrei.

\section{$\S 75$}

Die Kosten der Fürsorgeerziehung sind dem Kostenträger auf sein Verlangen aus dem pfändbaren Vermögen des Minderjährigen oder des auf Grund des Bürgerlichen Rechts zu seinem Unterhalt Verpflichteten zu erstatten. Die näheren Bestimmungen trifft die Landesgesetzgebung. Allgemeine Verwaltungskosten werden nicht ersetzt.

\section{$\S 76$}

(1) Wer, abgesehen von den Fällen der $\S \S 120,122 b$, 235 des Strafgesetzbuchs, einen Minderjährigen, bezüglich dessen das gerichtliche Verfahren auf Unterbringung zur Fürsorgeerziehung eingeleitet oder die Unterbringung zur Fürsorgeerziehung angeordnet ist, dem Verfahren oder der angeordneten Fürsorgeerziehung entzieht oder ihn verleitet, sich dem Verfahren oder der Fürsorgeerziehung zu entziehen, oder wer ihm hierzu vorsätzlich behilflich ist, wird auf Antrag der Fürsorgeerziehungsbehörde mit Gefängnis bis zu zwei Jahren und mit Geldstrafe oder mit einer dieser Strafen bestraft. Die Zurücknahme des Antrags ist zulässig.

(2) Der Versuch ist strafbar.

\section{Schlußbestimmungen}

\section{$\$ 77$}

Welche Behörden die in diesem Gesetze der obersten Landesbehörde oder dem Landesjugendamte übertragenen einzelnen Aufgaben wahrzunehmen haben, bestimmt die Landesregierung.

\section{§ $\mathbf{7 8}^{*}$ )}

-) aufgehoben durch vo v. 14. 2. 1924. 